\title{
L'hyperplasie bilatérale des processus coronoïdes (maladie de Langenbeck) : à propos d'un cas
}

\author{
Marion Canonica ${ }^{1, *}$, Philippe Lesclous ${ }^{2}$, Said Kimakhe ${ }^{3}$, Guy Princ ${ }^{4}$ \\ 1 Interne DESCO, Service de stomatologie et chirurgie maxillo-faciale, groupe hospitalier Paris Saint-Joseph, Paris, France ; Unité fonctionnelle \\ de chirurgie orale, service d'odontologie chirurgicale et restauratrice, CHU Hôtel Dieu de Nantes, faculté de chirurgie dentaire, Nantes, France \\ 2 PU-PH, Unité fonctionnelle de chirurgie orale, service d'odontologie chirurgicale et restauratrice, CHU Hôtel Dieu de Nantes, faculté de chirurgie \\ dentaire, Nantes, France \\ 3 MCU-PH, Unité fonctionnelle de chirurgie orale, service d'odontologie chirurgicale et restauratrice, CHU Hôtel Dieu de Nantes, \\ faculté de chirurgie dentaire, Nantes, France \\ 4 PH, Service de stomatologie et chirurgie maxillo-faciale, groupe hospitalier Paris Saint-Joseph, Paris, France \\ Correspondance : marion_ka@hotmail.com
}

(Reçu 21 février 2014, accepté 18 juillet 2015)

Mots clés :

processus coronoïde / maladie de Langenbeck / maladie de Jacob

Key words: coronoid process / Langenbeck's disease / Jacob's disease
Résumé - Introduction : L'hyperplasie bilatérale des processus coronoïdes ou maladie de Langenbeck est une pathologie rare caractérisée par une limitation non douloureuse de l'ouverture buccale. Observation : Un adolescent âgé de 16 ans avait consulté son praticien pour une limitation de l'ouverture buccale $(22 \mathrm{~mm})$ inexpliquée et persistante depuis 3 ans, sans lésion décelable à l'imagerie de première intention. Un diagnostic de dysfonctionnement méniscal bilatéral était posé et un traitement par gouttière occlusale et kinésithérapie entrepris sans résultats probants. Une imagerie par résonance magnétique et une tomodensitométrie étaient alors réalisées et mettaient en évidence une hyperplasie bilatérale des processus coronoïdes. Une intervention chirurgicale à type de coronoïdectomie bilatérale était réalisée. L'examen histologique des pièces opératoires confirmait la présence d'une hypertrophie osseuse sans anomalie structurale. Après 3 semaines de kinésithérapie, l'ouverture buccale était alors de $36 \mathrm{~mm}$. Discussion : La TDM 3D et l'histologie permettent de distinguer les différentes formes d'hyperplasie du processus coronoïde que sont la maladie de Langenbeck et celle de Jacob. La coronoïdectomie associée à de la kinésithérapie maxillo-faciale postopératoire est le traitement de choix. Conclusion : La rareté et la méconnaissance de ce type de pathologie conduisent souvent à des retards de diagnostic comme l'illustre le cas présenté.

Abstract - Bilateral hyperplasia of the coronoid processes (Langenbeck's disease): a case report. Introduction: Bilateral hyperplasia of the coronoid processes (Langenbeck's disease) is a rare entity characterized by a painless reduction of mouth opening. Observation: A 16-year-old patient was referred with a history of restriction of mouth opening during the last three years. The patient was diagnosed as having bilateral TMJ disorder and treated for this dysfunction without improvement. A MRI and orthopantomogram depicted normal joints bilaterally but without condylar movement associated with mouth opening. After his referral, a 3D CT scan revealed bilateral enlargement of the coronoid processes. Treatment involves bilateral coronoidectomy to relieve impingement on the zygoma. A histologic exam of the resected samples confirmed the diagnosis of bone hyperplasia. After three weeks of physical therapy, his mouth opening was $36 \mathrm{~mm}$. Discussion: The 3D CT scan and histologic exam allow one to distinguish between two forms of hyperplasia of the coronoid process: Langenbeck's disease (painless bilateral elongation of the coronoid processes) and Jacob's disease (unilateral osteochondroma). The treatment of choice is coronoidectomy in both cases. Conclusion: As illustrated in this case, due to its low prevalence, the diagnosis of this pathology is not straightforward.

\section{Introduction}

L'hyperplasie bilatérale des processus coronoïdes est une pathologie rare, décrite pour la première fois en 1853 par Langenbeck $[1,2]$. Elle est caractérisée par une limitation non douloureuse de l'ouverture buccale ; les processus coronoïdes hyperplasiques venant buter contre l'os zygomatique. Jacob, quant à lui, décrit en 1899 l'ostéochondrome du processus coronoïde responsable d'une constriction permanente des mâchoires et parfois d'une déformation faciale ; une néoarticulation se créant entre ce processus et l'os zygomatique qui se remanie $[1,2]$. Une certaine confusion peut exister entre ces 


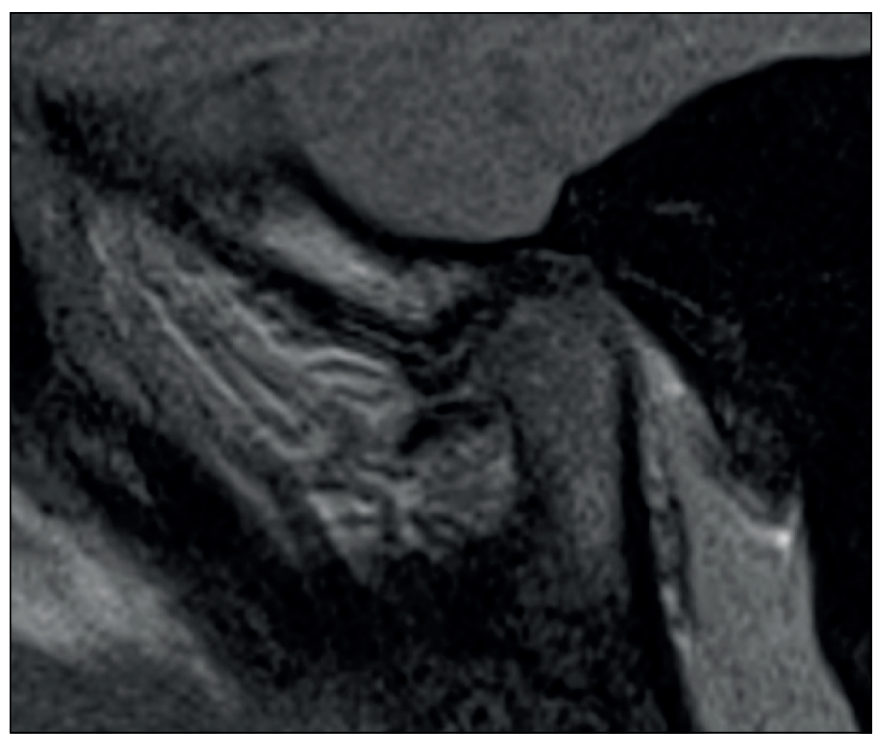

Fig. 1. IRM de l'ATM, bouche ouverte : déplacement méniscal antérieur. Fig. 1. MRI of the temporomandibular joint, open mouth: anterior disc displacement.

deux entités. Que cela soit la maladie de Jacob ou de Langenbeck, peu de cas sont décrits dans la littérature. Elles peuvent donc représenter un réel dilemme diagnostique.

\section{Observation clinique}

Un adolescent âgé de 16 ans avait consulté son chirurgiendentiste pour une limitation de l'ouverture buccale inexpliquée et persistante depuis trois ans. Cette limitation de l'ouverture buccale était présente depuis l'enfance d'après lui, associée à des bruits articulaires, temporo-mandibulaires. Il n'avait jamais consulté pour ce motif auparavant et il n'y avait aucune notion d'aggravation ni d'amélioration.

L'ouverture buccale, sans latérodéviation, était évaluée à $22 \mathrm{~mm}$. L'imagerie panoramique (OPT) et cone beam (CBCT) ne décelait aucune lésion osseuse des maxillaires. Devant le tableau clinique, le praticien posait le diagnostic de dysfonctionnement méniscal bilatéral. Il réalisait alors une gouttière occlusale et prescrivait 15 séances de kinésithérapie maxillofaciale.

Après deux mois de traitement, les bruits articulaires avaient disparu mais l'ouverture buccale n'avait augmenté que de 2 millimètres. Devant l'absence d'amélioration significative, une IRM des ATM était prescrite et le patient adressé à un service de chirurgie maxillo-faciale. L'interrogatoire de l'adolescent et de ses parents recherchait une cause de constriction permanente des mâchoires. Aucune pathologie générale ni aucun antécédent de traumatisme mandibulaire ou d'infections ORL (otites, mastoïdites à répétition) n'étaient retrouvés.

Le CBCT permettait de visualiser une limitation de la translation des condyles mandibulaires. À l'IRM, on retrouvait un déplacement limité des ménisques sans déformation méniscale (Fig. 1).

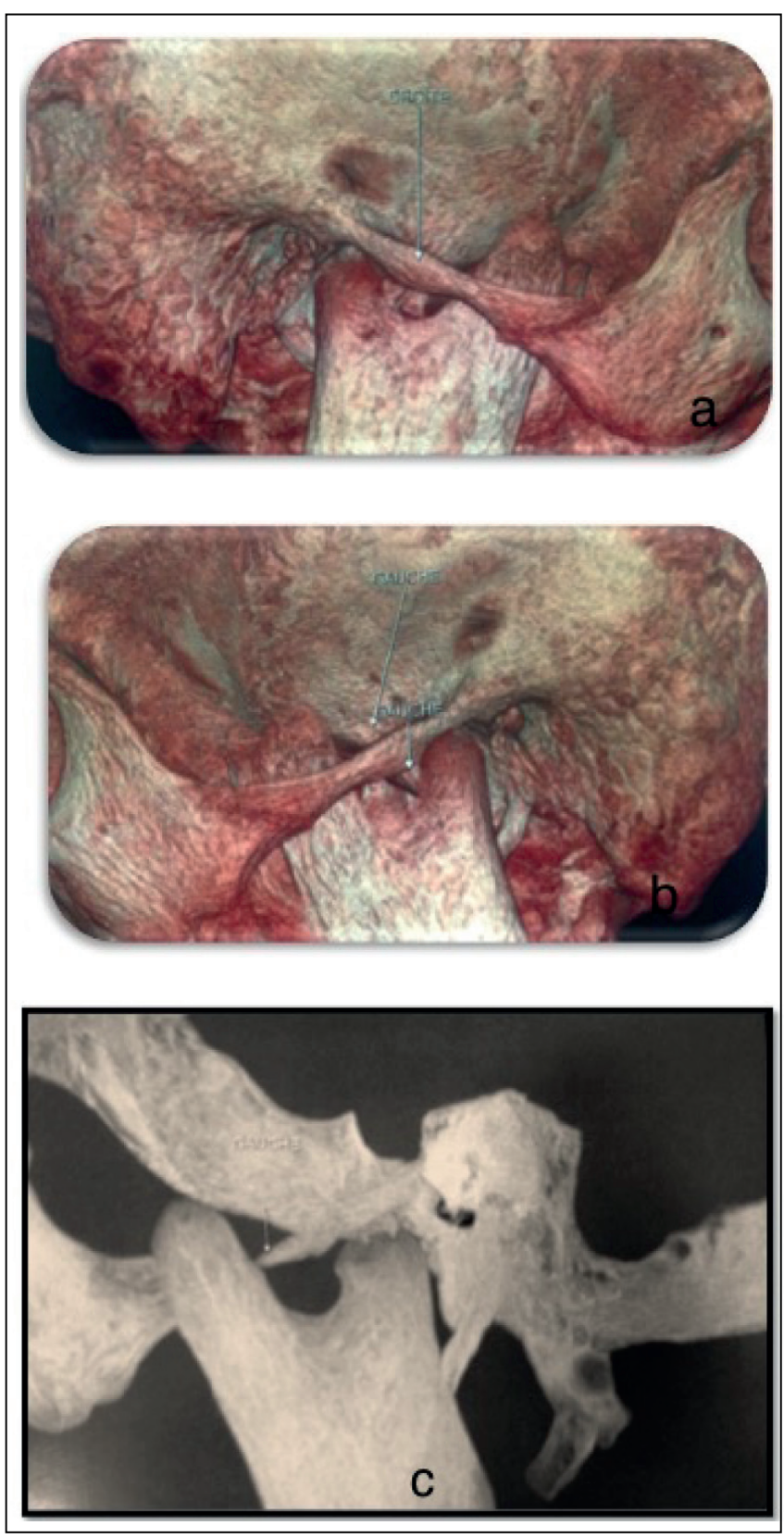

Fig. 2. TDM 3D : hyperplasie bilatérale des processus coronoïdes avec butée sur l'os zygomatique.

a) côté droit ; b) et c) côté gauche.

Fig. 2. 3D CT scan: the relationship between coronoid processes and the zygomatic bones is readily seen.

a) right side; b) and c) left side.

On ne pouvait donc conclure à une luxation discale irréductible. Devant la difficulté diagnostique, une tomodensitométrie (TDM) du massif facial avec reconstruction 3D était demandée. Cet examen mettait en évidence une hyperplasie bilatérale des processus coronoïdes entravant l'ouverture buccale (Fig. 2).

Une intervention chirurgicale à type de coronoïdectomie bilatérale était proposée; en insistant bien sur les limites de la 


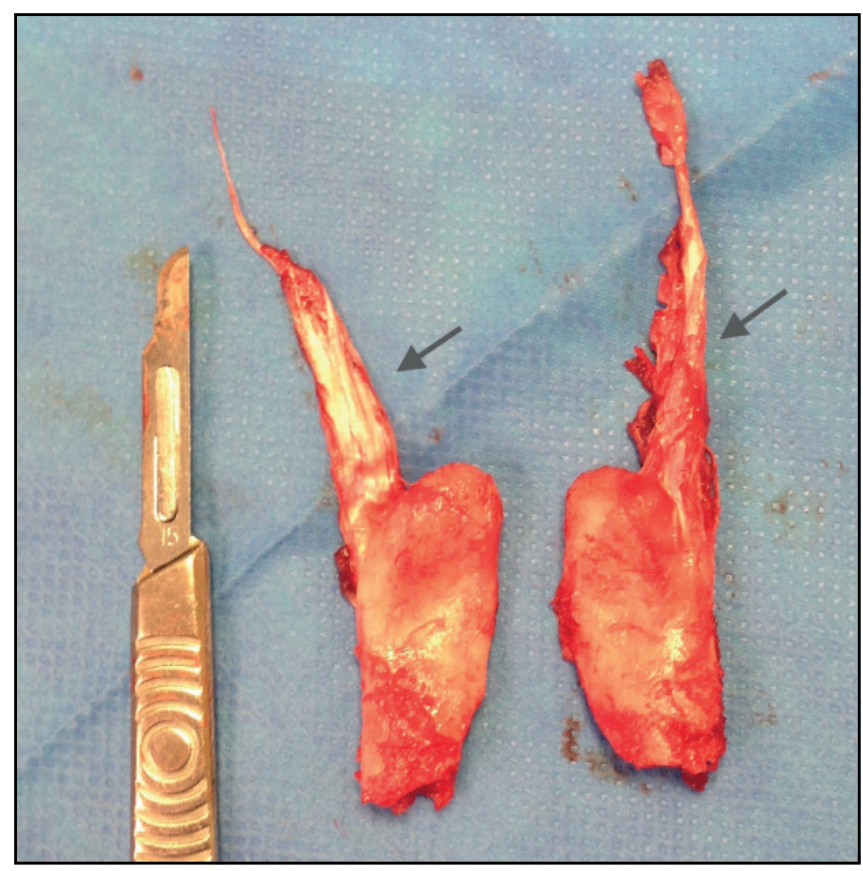

Fig. 3. Processus coronoÏdes réséqués avec attache du muscle temporal (flèche).

Fig. 3. Left and right coronoid process with the insertion of the temporalis muscle (arrow).

chirurgie c'est-à-dire la nécessité d'y associer une kinésithérapie maxillo-faciale permettant d'améliorer la dynamique.

La chirurgie avec une voie d'abord endobuccale avait eu lieu sous anesthésie générale avec une intubation nasotrachéale par fibroscopie (intubation difficile). L'ostéotomie des processus coronoïdes permettait leur résection (Fig. 3 et 4 ).

L'examen histologique des pièces opératoires confirmait la présence d'une hyperplasie osseuse sans anomalie structurale (Fig. 4). Les résultats postopératoires immédiats étaient peu concluants. Cependant, après trois semaines et plusieurs séances de kinésithérapie, l'ouverture buccale était alors de $36 \mathrm{~mm}$.

\section{Discussion}

L'hyperplasie des processus coronoïdes qu'elle soit unilatérale ou bilatérale est une cause rare de limitation de l'ouverture buccale. D'après Tavassol et al., une mesure normale sur scanner d'un processus coronoïde est d'environ 13,02 mm chez l'adulte contre 12,43 mm chez un adolescent [3]. L'étiologie n'est pas vraiment connue et les causes proposées sont : une hyperactivité des muscles temporaux, des troubles endocriniens, des anomalies génétiques ou des déplacements discaux chroniques [2].

L'OPT permet de suspecter l'hyperplasie mais seule la TDM avec reconstruction 3D pourra confirmer le diagnostic. L'IRM des ATM, souvent réalisée en première intention, ne permet pas de poser ce diagnostic ; les processus coronoïdes étant hors des champs de coupe sur ce type d'examen. La course du ménisque,

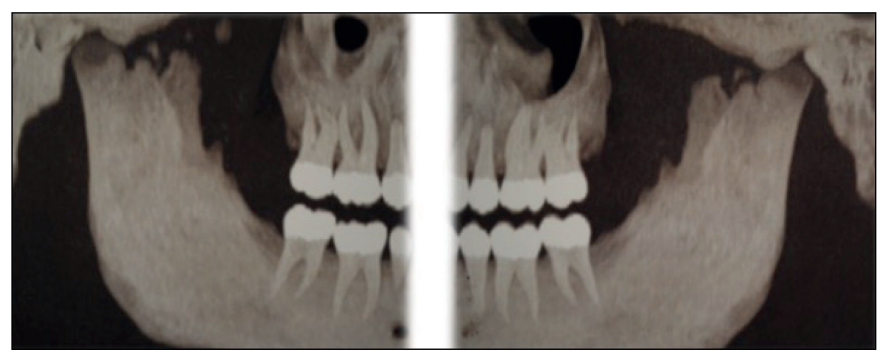

Fig. 4. TDM postopeératoire montrant les processus coronoÏdes réséqués.

Fig. 4. Postoperative CT scan with the resected coronoid processes.

limitée du fait de la limitation de l'ouverture buccale, est d'interprétation délicate.

L'analyse de la littérature révèle deux entités différentes d'élongation du processus coronoïde. La maladie de Jacob correspond à une néoarticulation entre un ostéochondrome du processus coronoïde et l'arcade zygomatique remaniée. Dans la majorité des cas, l'anomalie est unilatérale et peut provoquer une asymétrie faciale (déformation unilatérale de l'os zygomatique). Des douleurs peuvent être présentes. L'ostéochondrome est une tumeur bénigne que l'on retrouve rarement au niveau maxillo-facial. La confirmation de ce diagnostic se fait avec l'examen histologique [1-4]. Une cinquantaine de cas sont répertoriés dans la littérature.

La deuxième entité, comme celle décrite dans le cas présent, est une élongation du processus coronoïde sans anomalie osseuse. Encore appelée maladie de Langenbeck, elle est généralement bilatérale. Environ 80 cas ont été rapportés mais l'incidence de cette pathologie doit être bien plus élevée ; l'absence de douleur n'amenant que rarement à consulter comme dans le cas présent $[3,5,6]$. D'ailleurs, Hönig et al., en 1993, estiment à 0,5\% la fréquence d'hyperplasie du processus coronoïde dans la population générale [7].

La TDM 3D et l'histologie permettent de distinguer les différentes formes d'hyperplasie du processus coronoïde. La coronoïdectomie associée à de la kinésithérapie maxillo-faciale postopératoire est le traitement de choix. La récidive est rare. L'abord chirurgical peut être intra-oral, extra-oral ou mixte. L'intervention endobuccale évite les cicatrices disgracieuses et les risques de léser le nerf facial. Cependant, cette voie n'est pas toujours possible en cas de trismus sévère et dans le cas de la maladie de Jacob, elle peut être limitée par la taille de l'exostose $[2,4-6,8]$.

\section{Conclusion}

La rareté et la méconnaissance de ce type de pathologie conduisent souvent à des retards de diagnostic comme l'illustre le cas présenté. L'hyperplasie des processus coronoïdes n'est pas toujours visible sur un OPT et le diagnostic est rendu plus facile par l'examen TDM avec reconstruction 3D.

Conflits d'intérêt : aucun 


\section{A S C L I N I Q U E}

\section{Références}

1. D'Ambrosio N, Kellman RM, Karimi S. Osteochondroma of the coronoid process (Jacob's disease): an unusual cause of restricted jaw motion. Am J Otolaryngol 2011;32:52-54.

2. Coll-Anglada M, Acero-Sanz J, Vila-Mansana I, NavarroCuéllar C, Ochandiano-Caycoia S, López de-Atalaya J, et al. Jacob's disease secondary to coronoid process osteochondroma. A case report. Med Oral Patol Oral Cir Bucal 2011;16:e708-710.

3. Tavassol F, Spalthoff S, Essig H, Bredt M, Gellrich NC, Kokemüller $\mathrm{H}$. Elongated coronoid process: CT-based quantitative analysis of the coronoid process and review of literature. Int J Oral Maxillofac Surg 2012;41:331-338.

4. Sreeramaneni SK, Chakravarthi PS, Krishna Prasad L, Raja Satish P, Beeram RK. Jacob's disease: report of a rare case and literature review. Int J Oral Maxillofac Surg 2011;40:753-757.

5. Satoh K, Ohno S, Aizawa T, Imamura M, Mizutani H. Bilateral coronoid hyperplasia in an adolescent: report of a case and review of the literature. J Oral Maxillofac Surg 2006;64:334-338.

6. McLoughlin PM, Hopper C, Bowley NB. Hyperplasia of the mandibular coronoid process: an analysis of 31 cases and a review of the literature. J Oral Maxillofac Surg 1995; 53:250-255.

7. Hönig JF, Merten HA, Halling F, Korth OE. Rötgenologische studie zur häufigkeit der asymptomatischen processuscoronoideus-vergröberung. Schweiz Monatschr Zahnheilkund 1993;103:281-284.

8. Gagé J, Gallucci A, Stroumsa R, Foletti JM, Guyot L, Chossegros C. Transoral coronoidectomy: technical note. Rev Stomatol Chir Maxillofac Chir Orale 2015;116(6): 368-371. 